\title{
XLIII. The comparison of capacities in electrical work; an application of radioactive substances
}

\section{J.A. McClelland M.A.}

To cite this article: J.A. McClelland M.A. (1904) XLIII. The comparison of capacities in electrical work; an application of radioactive substances, Philosophical Magazine Series 6, 7:40, 362-371, DOI: $10.1080 / 14786440409463128$

To link to this article: http://dx.doi.org/10.1080/14786440409463128

册 Published online: 15 Apr 2009.

Submit your article to this journal $\lceil\pi$

Џ Article views: 3

Q View related articles $₫$ 
the radium atom breaks up. The radium atom certainly gives off positively charged particles-the $a$ rays. The emanation particles cannot be what remains of the atom after the emission of one or more a rays, because, in that case, it would be negatively charged. The atom must have parted with an equal negative charge, either by the emission of negative particles or in some other way.

XLIII. The Comparison of Capacities in Electrical Work; an Application of Radioactive Substances. By J. A. MoClelland, M.A., Professor of Experimental Physics, University College, Dublin*.

THERE are many methods by which two capacities may be compared, and which are fully described in textbooks of Physies.

When only approximate results are required, we have several methods to choose from, any of which will give a fair result; but the problem is by no means so simple when an accurate determination is required, especially if we are dealing with a very small capacity. That better methods of dealing with the determination of capacities, especially small capacities, are still required, may be judged from the fact that two papers have recently appeared on the subject, one by Professor Fleming and Mr. Clinton in the Phil. Mag., May 1903, and the other by Professor Stroud and Mr. Oates in the Phil. Mag., December 1903.

Those two papers may be taken as affording examples of the difficulty of obtaining accurate results in this work, both methods necessitating somewhat elaborate apparatus, and involving considerable experimental difficulties.

My object in this paper is to describe a method at once simple and accurate, and suitable for the determination of capacities of any magnitude down to a few micro-microfarads, or even less. The method is based on the fact that the ionization current that can be obtained by the use of a radioactive substance like uranium is extremely constant, and can be made so small that the time taken to charge a condenser by it can be accurately measured. This small constant current is used first to charge one condenser to a given potential ; and then a second condenser is charged to the same potential, and the time taken in the two cases observed, so that we get

* From an advance prouf of the Proceedings of the Royal Dublin Society, vol. x. part ii. p. 167, communicated by the Author. 
the ratio of two capacities by simply observing two interrals of time.

The method will probably have occurred to any one who has been using radioactive substances ; but as many workers have occasion to compare capacities accurately who are not using radioactive substances, I have thought it advisable to make a few experiments showing the accuracy of the method, and showing also how small a capacity can be detected and measured by it.

To use the method it is not necessary to hare a supply of radium, as the title of the paper might suggest ; uranium is even better in some respects, and uranium is to be found in every laboratory.

\section{Description of Apparatus and Method of Working.}

$A$ and $B$ are two insulated metal plates, one of which, B, can be joined to one terminal of a battery of small storage-cells, the other terminal of which is to enth. The battery may consist of 100 or more small test-tube cells, so that $B$ can be lept at 200 volts or higher.

Fig. 1.

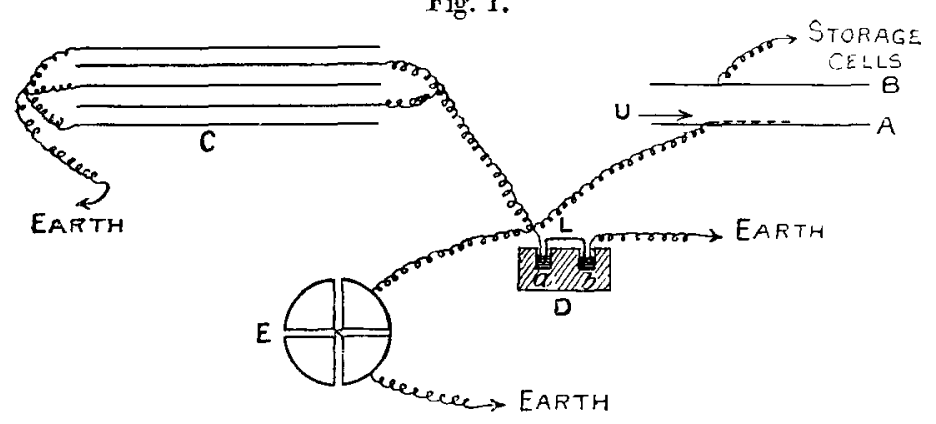

A few grammes of, say, uranium nitrate are spreal on a sheet of paper, and placed on the plate $A$. The radiation from the uranium ionizes the air between $A$ and $B$; and $: 0 A$ gradually rises in potential if insulated, supposing $\mathrm{B}$ to be positive. As is well known, the ionization current thus obtained between two plates increases at first as the potentialdifference between the plates increases; but when this potential-difference is made sufficiently great, the current attains a maximum, and does not further increase for further increase of potential-difference between the plates. If then $B$ is kept at a sufficiently high potential, small changes in this potential, due to the potential of the battery falling, will produce no effect; and, again, in making an observation, the potential of 
A need never change by more than one volt, so that there is no trouble in keeping a constant current to the plate $A$. The constancy of the current in the above arrangement only depends on the constancy of the radiation from the uranium; and numbers will be given to show how very constant this radiation is. The potential-difference required to produce the maximum current to $A$ will depend on the distance between $A$ and $B$; but it is well to have 200 volts available.

$\mathrm{C}$ represents one of the condensers being compared; and $\mathrm{E}$ is a quadrant-electrometer. $\mathrm{D}$ is an insulating block of paraffin, containing two mercury cups, $a$ and $b$, one of which is connected to earth. A connecting piece $L$ is shown, joining the mercury cups, and by means of a string arrangement $\mathrm{L}_{\mathrm{c}}$ can be lifted out of the mercury cups and lowered again as desired, from a distance, so as to avoid induction effects produced by movements of the observer.

As soon as $L$ is lifted out of the mercury cups, the plate $A$, the condenser $\mathrm{C}$, and the electrometer begin to charge up; and the time is observed during which the spot of light moves over, say, 100 scale-divisions. An exactly similar experiment is done with a second condenser $\mathrm{C}^{\prime}$ in the place of $\mathrm{C}$. If the intervals of time are respectively $t$ and $t^{\prime}$, we have

$$
\frac{\mathrm{C}+c}{\mathrm{C}^{\prime}+c}=\frac{t}{t^{\prime}},
$$

where $c$ is the capacity of the electrometer, the condenser $\mathrm{AB}$, and the connecting wires. The capacity $c$ can be determined in the terms of, say, $\mathrm{C}$, by taking an observation with $\mathrm{C}$ joined up as shown, and then an observation with $\mathrm{C}$ disconnected, so that only $c$ is charged ; or the capacity $c$ may bo determined once for all by comparing it in this way with a known capacity. We thus get the ratio $\mathrm{C} / \mathrm{C}^{\prime}$.

\section{Aceuracy of the Method.}

'The accuracy of the method obviously depends simply on the constancy of the ionization current, and on the accuracy with which the time-intervals are measured. Numbers are given below to show how very constant the ionization current is. In practice it is well to screen the space between the plates A and $\mathrm{B}$ from air-currents, as such currents, if strong, may blow away the ionized air, and diminish the ionization-current. As regards the radioactive substances used, uranium is preferable to thorium or radium, as it gives off no emanation. If radium or thorium is used, it should be in a closed vessel to prevent the emanation from escaping, otherwise the ionization-current will not be steady. 
The method involves the use of a quadrant-electrometer, which to some may appear an objection to the method. The writer's experience, however, is that no sensitive scientific instrument gives less trouble in working than a quadrantelectrometer, when it has once been put in good order. When the capacities being compared are small, no great sensitiveness of the electrometer will be required-say 60 millimetre scale-divisions for one volt with scale one metre from electrometer. When large capacities are being compared greater sensitiveness will be necessary, unless a very large quantity of uranium is used; but there is no trouble in having an instrument one hundred times as sensitive as above.

\section{Some Experiments with this Metlod.}

(a) We shall first give some numbers to show the constancy of the ionization-current in the above arrangement, and the accuracy with which the time required to charge any system through a given range of potential can be measured. 'The system charged consisted of the electrometer, a capacity marked $\bullet 01$ microfarad, and the condenser formed of the plates between which the uranium is placed.

The time taken for the spot of light to move over fifty scale-divisions was taken with a stop-watch reading to fifthis of a second. A series of seven observations was made, giving the following numbers, no observation being rejected.

Time taken to move over 50 scale-divisions :-

$$
\begin{array}{cc}
99 \cdot 2 & \text { seconds. } \\
99 \cdot 5 & " \\
99 \cdot 0 & " \\
99 \cdot 4 & , \\
99 \cdot 2 & " \\
99 \cdot 2 & " \\
99 \cdot 1 & " \\
\text { Mean... } & 99 \cdot 23 \text { seconds. }
\end{array}
$$

The agreement between these numbers is no better than that usually observed in other experiments; in fact, not as good as in many other cases.

(b) We shall now give the numbers observed in a comparison of a condenser with a standard condenser, marked -001 microfarad. We shall denote the capacity of the condenser to be measured by $\mathrm{C}$, and the capacity of the electrometer and other parts of the system by $c$.

The electrometer in this experiment gave a deflexion of Plil. Mag. S. 6. Vol. 7. No. 10. April 1904. 2 C 
about 60 scale-divisions for 1 volt, and about 30 grams of nranium nitrate were placed on the central part of the plate $\mathbf{A}$; observations were taken alternately with the capacity $\mathrm{C}$ joined up to $c$, and with the capacity 001 microfarad joined up to $c$, giving results as follows:-

$$
\cdot 001+c \text {. }
$$

50 divisions in $52 \cdot 6$ seconds

50 divisions in $52 \cdot 5$,

50 divisions in $52 \cdot 2$,

Mean... 52.43"

$$
\mathrm{C}+c \text {. }
$$

50 divisions in 89.5 seconds 50 divisions in 88.7, Mean... $89 \cdot 1$,

Therefore

$$
\frac{\mathrm{C}+c}{.001+c}=\frac{8910}{5243} \text {. }
$$

A smallex quantity of uranium was then used to determine. the ratio between $c$ and $\cdot 001$ microfarad, as with the quantity used above the movement of the spot of light would have been too rapid when only the capacity $c$ was in use. The following are the numbers in this determination :-

$$
\text { c+. } 001 \text {. }
$$

$c$.

50 divisions in 75.8 seconds 50 divisions in $75 \cdot 7$,

100 divisions in 21.9 seconds. 100 divisions in $21 \cdot 8 \quad$, 100 divisions in $22 \cdot 2$,

$$
\text { Mean... 75.75, }
$$$$
\text { Mean... 21.97, }
$$

Therefore

$$
\frac{c+\cdot 001}{c}=\frac{15150}{2197} .
$$

These equations give

$$
\begin{aligned}
& e=\cdot 000169 \text { microfarad; } \\
& \mathrm{C}=\cdot 001817 \text { microfarad }
\end{aligned}
$$

To give somewhat of a test of the reliability of the method, the same capacity was determined on another occasion, taking no care to use the same quantity of uranium, and, in fact, having very different ionization-currents from those used in the first case. The following numbers were obtained, only one observation being taken in each case :-

$$
\mathrm{C}+c \text {. }
$$

50 divisions in $48 \cdot 2$ seconds

$$
\text { ot.001. }
$$

50 divisions in 56.5 $.001+c$.

50 divisions in 28.6 seconds

$c$.

100 divisions in $16^{\circ} 5$, 
Calculating as before, we get

$$
\begin{aligned}
& c=\cdot 000170 \text { microfarad, } \\
& \mathrm{C}=\cdot 001802 \text { mierofarad. }
\end{aligned}
$$

The agreement with the preceding numbers is very good, especially when we consider that only one observation was taken in each case.

(c) To show that this method is suitable for much larger capacities than those used in the preceding examples, we shall give an example in which a capacity known to be about .5 microfarad was determined by comparing it with a standard capacity of 1 microfarad. For this purpose, an electrometer of the Dolezalek type was used, giving a deflexion equal to 5300 scale-divisions for 1 volt.

Observations were taken as before, first with the unknown capacity $\mathrm{C}$ joined up to the elestrometer and the plate $\mathrm{A}$, and then with the capacity $\cdot 1$ microfarad joined up.

About 100 grams of uranium nitrate were placed on $\mathrm{A}$ (fig. 1), and the following numbers noted; $c$ denotes the capacity of the Dolezalek electrometer, and some apparatus that was in connexion with it:-

$$
\mathrm{C}+c . \quad \cdot 1+c .
$$

50 divisions in 104.5 seconds 100 divisions in 41.8 second: 50 divisions in 104.4 " 100 divisions in 41.8 .,

$$
\text { Mean... 104.45 ". Mean... 41.8 , }
$$

Therefore

$$
\frac{C+c}{1+c}=\frac{208 \cdot 9}{41 \cdot 6}
$$

Less than 1 gram of uranium nitrate was now used to determine the ratio of $c$ to a known capacity of $\cdot 001$ microfarad, giving as follows :-

$$
\text { c. } \quad c+001 \text {. }
$$

100 divisions in 21.0 seconds 100 divisions in 30.5 seconds 100 divisions in $21 \cdot 2, \quad 100$ divisions in $30^{\circ} 0$,

Therefore

$$
\frac{c+\cdot 001}{t}=\frac{605}{42 \%}
$$

These equations give-

$$
\begin{aligned}
& c=\cdot 0023 \text { microfarad, } \\
& \mathrm{C}=.5089 \text { microfarad. }
\end{aligned}
$$

A repetition of this determination gave as before, with smaller capacities, an equally consistent result. 
(d) A careful experiment was now made to find how small a capacity could be detected and measured by this method.

To do this a condenser of the following type (fig. 2) was arranged.

Fig. 2.

\section{$B$}

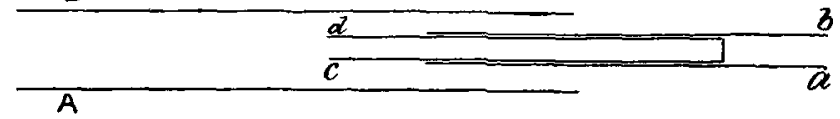

$\mathrm{AB}$ is a long wide tube, $7.90 \mathrm{cms}$. internal diameter ; $a b$ is another tube fixed, as shown, to be coaxial with $A B$. In $a b$ a third cylinder $c d$ slides, fitting closely into $a b$, the external diameter of $c d$ being $1 \cdot 94 \mathrm{~cm}, \mathrm{AB}$ is joined to earth, and $a b$ (and $c d$ ) connected to the Kolvin electrometer. The capacity of the electrometer, the condenser as arranged (fig. 2), and joining wires is determined by comparing it with a standard capacity of $\cdot 001$ microfarad.

A careful series of observations is then taken, with $c d$ in its above position, using a suitable quantity of uranium nitrate. Then the tube $c d$ is moved $8.01 \mathrm{cms}$. further into $\mathrm{AB}$, care being taken not to displace $\mathrm{AB}$ or $a b$. A vernier was attached to $c d$ working in a slot in $a b$, so that the distance through which $c d$ was displaced could be accurately measured. A second series of observations was then made with $c d$ in the new position, keeping the same uranium as before. We have thus the data for deducing from the experiments the increase of capacity produced by the movement of $c d$. This increase of capacity can also be accurately calculated from the formula

$$
\frac{l}{2 \log \frac{r_{1}}{r_{2}}}
$$

since the effects of the ends are eliminated by the arrangement used, $l$ being the distance $c d$ is moved, and $r_{1}$ and $r_{2}$ the radii of $\mathrm{AB}$ and $c d$ respectively. We can thus estimate the value of the method for measuring very small capacities.

The numbers observed were as follows:-

(1) Finding the capacity $\mathrm{C}$ made up of the condenser described (fig. 2), the electrometer, and connexions.

C.

100 divisions in $39 \cdot 7$ seconds 100 divisions in $39 \cdot 6$ 100 divisions in $39 \cdot 5$ Mean... $39 \cdot 6$,

$$
001+\mathrm{C} \text {. }
$$

50 divisions in $117 \cdot 8$ seconds 50 divisions in $118 \cdot 1$, 50 divisions in 117.8 ", Mean... 117.9, 
of Capacities in Electrical Work.

Therefore

$$
\frac{\mathrm{C}+\cdot 001}{\mathrm{C}}=\frac{235 \cdot 8}{39 \cdot 6}
$$

$\begin{array}{ll}\text { or } & \mathrm{C}=\cdot 000201 \text { microfurad; } \\ \text { or } & \mathrm{C}=201 \text { micro-microfarads. }\end{array}$

(2) To find the small change in the capacity $\alpha$ when $c d$ has been mored into its second position.

In first position :-

$$
\begin{gathered}
100 \text { divisions in } 59 \cdot 0 \text { seconds, } \\
100 \text { divisions in } 59 \cdot 5, \\
100 \text { divisions in } 59 \cdot 1, \\
\text { Mean... } 59 \cdot 20,
\end{gathered}
$$

In second position :-

100 divisions in 60.5 seconds,

100 divisions in $59 \cdot 8$,

100 divisions in 60.0

$$
\text { Mean... 60.10, }
$$

Therefore

$$
\frac{\mathrm{C}+\alpha}{\mathrm{C}}=\frac{60 \cdot 1}{59 \cdot 2}
$$

and

$$
\begin{aligned}
\quad \mathrm{C} & =201 \text { micro-microfarads; } \\
\therefore \quad a & =3 \cdot 05 \text { micro-microfarads. }
\end{aligned}
$$

Calculating $\alpha$ from the formula

where

$$
\alpha=\frac{l}{2 \log \frac{r_{1}}{r_{2}},}
$$

$$
\begin{aligned}
l & =8.01 \mathrm{cms}, \\
r_{1} & =3.95 \mathrm{cms}, \\
r_{2} & =.97 \mathrm{~cm} .
\end{aligned}
$$

we get

$$
\alpha=2.85 \text { electrostatic units }
$$$$
=3 \cdot 16 \text { micro-microfarads. }
$$

We get, therefore, $3 \cdot 16$ by calculation, and $3 \cdot 05$ by experiment.

The method is therefore quite capable of detecting and measuring with considerable accuracy a capacity of 1 micromicrofarad or even less. 
Discussion of the Advantages of the Method.

It is not necessary to compare this method in detail with the many other methods used in comparing capacities; it will be sufficient to point out a few leading facts.

Capacities may, of course, be compared by the electrometer without any use of radioactive substances by charging the unknown capacity to a potential which is measured by the electrometer, and then sharing the charge with a known capacity, and again measuring the potential. The method of working is not, however, as accurate as that described above, especially when the capacities are small.

Capacities are often compared by charging them to the same potential, and discharging them through a ballistic galvanometer. The galvanometer-deflexion must be accurately read, and a correction applied for damping, observations which cannot be made with the accuracy with which we can compare two intervals of time. Again, when the capacities are small, they must be charged or discharged through the galvanometer a great number of times per second, which requires carefully constructed apparatus to enable the number of charges to be accurately known. In addition it is somewhat difficult to be certain that the apparatus is working properly; for example, an error might arise through faulty insulation, and escape detection.

The method of De Sauty is free from many of the objections mentioned above ; but others might be urged against it, and especially that it can be of little use when the capacities are very small.

One of the chief advantages of the method described in this paper is that, from the nature of the apparatus used, it is scarcely possible for any serious source of error to come in without detection ; a faulty insulation, for example, can easily be guarded against. The only quantity requiring to be measured is an interval of time, which can be done with great accuracy. The ionization produced by the uranium keeps very constant throughout the time required to make a determination, and there is no other quantity that requires to be kept very constant. The potential of the battery joined to one of the plates between which the uranium is placed may vary considerably between the observations and produce no effect, provided the potential is sufficiently great.

The only objection that seems likely to be made to the method is the fact that it employs a quadrant-electrometer, the use of which in ordinary laboratory work has hitherto been discouraged. As stated above, the writer sees no reason for the reluctance to use electrometers when their use can be 
avoided by means of galvanometers and other, sometimes complicated, apparatus. Some of the lines of research in recent years have necessitated an extensive use of quadrant electrometers, with the natural result that they have been greatly improved; and whatever reasons there may formerly have been for avoiding the use of electrometers, these reasons have now entirely disappeared.

\section{On a New Form of Sensitive Hot-Wire Voltmeter.} By R. Threlfall, F.R.S.*

TTHE practical need for a sensitive altemate-current roltmeter arises in connexion with the measurement of large alternating currents. 'The instruments at present employed for the purpose of measuring alternating currents are substantially of two types, - the ampere gange of Lord Kelvin forming the standard and almost only representative of one class; and instruments based on transformers forming the other. Botli classes of instruments require calibration in manufacture, and from time to time, and it is then that the want of a sensitive voltmeter is felt; for the obvionsly most direct method is to measure the potential drop across a standard resistance traversed by the whole current in question. It may seem curious that the larger the current to be measured, the more sensitive must be the roltmeter employed; but a little consideration will show that this is the case because it is not practicable to go on increasing the weight of a standard resistance without limit. In the other alternative the heating becomes excessive, and there is a risk of damaging the standard. For instance, suppose that it is a question of measuring 2000 amperes by means of a resistance of $\cdot 0002 \mathrm{ohm}$, the P.D. drop is 4 volt, and the power expended in heat is 8 kilowatt-quite a consideration. The practical standard alternating-current roltmeter must therefore be sensitive and adjusted to work across an external resistance which may be considered negligible in comparison with its own resistance. If the hot-wire form be adopted, it is seen that the electrical considerations point to the wire being as short as possible, and also as tine as possible; for it has often been shown that the rise of temperature for a giren current-density increases as the diameter of the wire decreases.

Taking everything into account, the most suitable material appears to be pure silver. As this can be obtained commercially nicely gilded and of such a thickness that two miles

* Communicated by the Physical Society : read November 27, 190:3. 